\title{
Crops diseases detection and solution system
}

\author{
Mohammad Jahangir Alam, Md. Abdul Awal, Md. Nurul Mustafa \\ Department of Computer Science and Information Technology, Southern University Bangladesh, \\ 739/A Mehidibag Road, Chittagong, Bangladesh
}

\begin{tabular}{l}
\hline \hline Article Info \\
\hline Article history: \\
Received Feb 14, 2018 \\
Revised Dec 17, 2018 \\
Accepted Jan 11, 2019 \\
\hline Keywords: \\
Agricultural experts \\
Android apps \\
CDDASS \\
Crops siseases detection \\
Image processing \\
\hline
\end{tabular}

\begin{abstract}
The technology based modern agriculture industries are today's requirement in every part of agriculture in Bangladesh. In this technology, the disease of plants is precisely controlled. Due to the variable atmospheric circumstances these conditions sometimes the farmer doesn't know what type of disease on the plant and which type of medicine provide them to avoid diseases. This research developed for crops diseases detection and to provide solutions by using image processing techniques. We have used Android Studio to develop the system. The crops diseases detection and solution system is compared the image of affected crops with database of CDDASS (Crops Diseases Detection and Solution system). If CDDASS detect any disease symptom, then provide suggestion so that farmers can take proper decision to provide medicine to the affected crops. The application has developed with user friendly features so that farmers can use it easily.
\end{abstract}

Copyright $\odot 2019$ Institute of Advanced Engineering and Science. All rights reserved.

\section{Corresponding Author:}

Mohammad Jahangir Alam,

Department of Computer Science and Information Technology,

Southern University Bangladesh,

739/A Mehidibag Road, Chittagong, Bangladesh.

E-mail: jahangir@southern.edu.bd

\section{INTRODUCTION}

Agriculture and human social development go side by side as the production of crops made it possible for primitive man to settle down in selected spots leading to formation of society [1]. The history of agricultural in Bangladesh long before. Bangladesh is basically an agricultural country, and the income is based on the agricultural products and all its resources depend on the agricultural output.

Although Bangladesh is on course for Middle Income Country status by 2021, agriculture remains the largest employer in the country by far and $47.5 \%$ of the population is directly employed in agriculture and around $70 \%$ depends on agriculture in one form or another for their livelihood. Agriculture is the source of food for people through crops, livestock, fisheries; the source of raw materials for industry, of timber for construction; and a generator of foreign exchange for the country through the export of agricultural commodities, whether raw or processed. It is the motor of the development of the agro-industrial sector including food processing, input production and marketing, and related services. As main source of economic linkages in rural areas, it plays a fundamental role in reducing poverty which remains a predominantly rural phenomenon [2].

Though in Agricultural Department technology is rapidly changing, many automatic technologies are coming in the market (example, Automatic planting, cutter machines etc which helps the farmer to produce maximum products). Plant disease is an important concern for the farmers in Bangladesh because Plant disease is an impairment of the normal state of the plant that interrupts or modifies its vital functions [3]. To get solution on planet disease if farmers decide to take advice from agricultural expert regarding the treatment of incidence of pest /disease/trait to their crop/plant in order to increase the crop productivity then he may face following problems [4]:

a. Sometimes they have to go long distances for approaching the expert. 
b. Even though they go such distances expert may not be available at that time.

c. Sometimes, the expert whom a farmer contacts, may not be in a position to advise the farmer with the available information and knowledge.

In these cases seeking the expert advice is very expensive and time consuming. To solve the above mentioned problems electronic expert systems are needed. Electronic expert systems enable farmers in identifying type of diseases; making the right decision and selecting the proper treatment. The expert systems are intelligent computer programs that are capable of offering solutions or advices related to specific problems in given domain, both in a way and at a level comparable to that of human expert in a field. One of the advantages of using Electronic expert systems is its ability to reduce the information that human users need to process, reduce personnel costs and increase throughput. Another advantage of expert system is that it performs tasks more consistently than human experts [5].

There are various kinds of abnormality states present on the plants leaf which can be identified by mean of manual inspection. The image processing and pattern recognition techniques play the worth full role to convert manual process to automate the process. The automatic diagnosis system based on plant disease features reduces the dependency on experts in the area concerned [6]-[8]. Depending on the application, many of those problems may be solved or at least reduced by the use of image processing.

Hence we are proposing an automatic crops diseases detection and solution system (CDDASS) which helps a farmer to identify the disease of crops and provide treatment for his crops according to disease using image processing techniques without help of any crops diseases expert. To get solution, farmers just need to install the apps into their mobile phones for the first time and upload the affected crops' image to detect and get suggestion from the system. That's way a farmer can save time, efforts and money and can be tension free.

\section{LITERATURE REVIEW}

Prof.H.M.Deshmukh, JadhavSanjivani, LoharUtkarsha, BhagatMadhuri and SalunkeShubhangi. "Plant Leaf Disease Identification System for Android". International Journal of Advanced Research in Computer and Communication Engineering [9]. In this article they have described the development of an Android application that gives users or farmers the capability to identify the plantleaf diseases based onthe photographs of plant leaves taken through an android application.

Sufiyan K Shekh, Aniket Baitule, Milind Narethe, Sangappa Mallad, Waghdarikar and Dr. D Y Patil. "Detection of Leaf Diseases and Monitoring the Agricultural Resources using Android App." International Journal of Innovative Research in Computer and Communication Engineering [10]. In this article they have described and designed a new architecture for remote control of agriculture devices and also detecting the diseases of plant which make much easier and less dependent of the conditions present to farmers.

Mr. Sushant. S. Chavan, Mr. Nitesh. P. Satre, Mr. Rajat. R. Deshmane, Mr. Suhas. B. Katkar "Android Based App to Prevent Crop Diseased in Various Seasons." International Research Journal of Engineering and Technology [11]. In this article they have developed an android application for agriculture, when and which fertilizers, pesticides and herbicides are used to save various crops from various diseases.

\section{PROPOSED METHOD}

\subsection{Flow chart}

The flowchart (Figure 1) describes the whole process of the research. At first user has to upload a defected crops image on the system then system will search image related information in the system database, if match the uploaded image with the system database it will provide possible diseases name with solution, if does not match it will show not match message. 


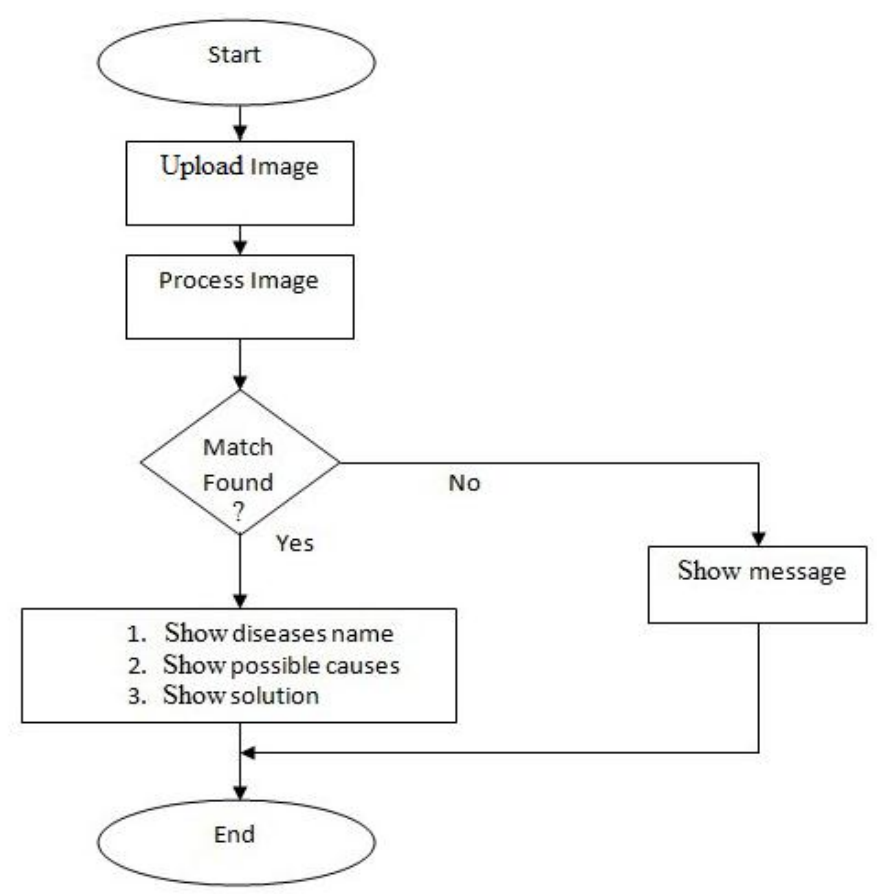

Figure 1. Flowchart of crops diseases detection and solution system

\subsection{Interface design}

The Figure 2 shows main page of the system where users can choose their activities including menu access and different crops list. A user can be able to see some diseases list with image as Figure 3 .

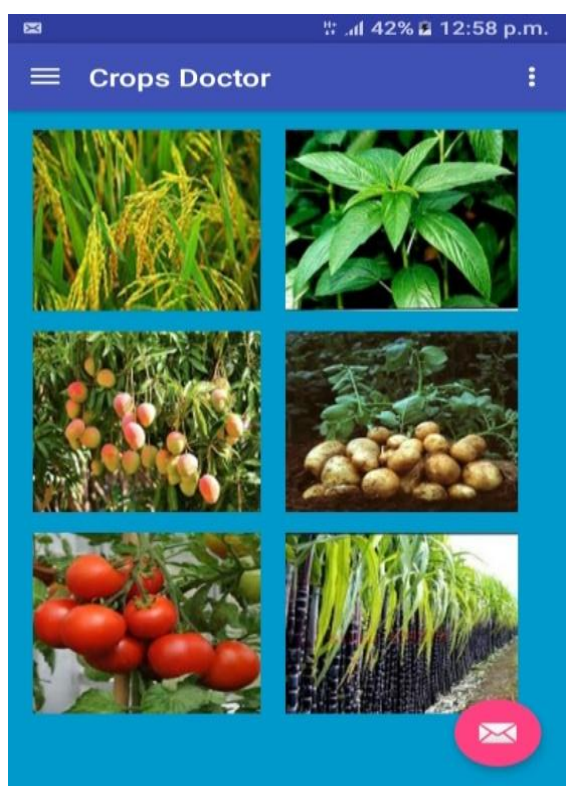

Figure 2. Home page of crops diseases detection and solution system

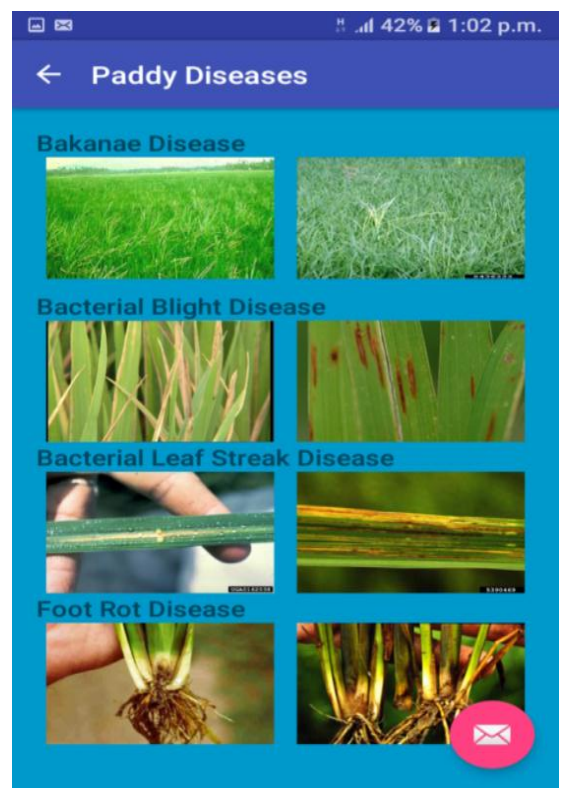

Figure 3. Diseases list page

After clicking any icon, image of diseases, visitor can be able to find specific disease symptoms with solutions like Figure 4. 


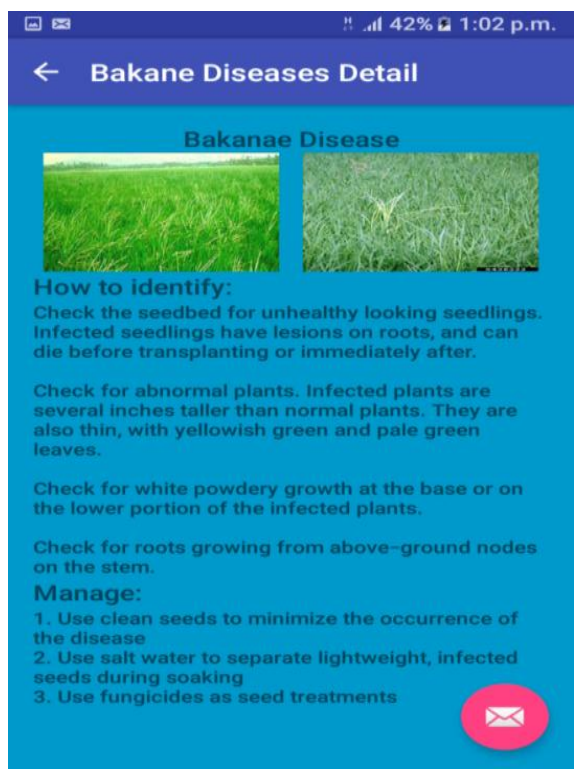

Figure 4. Disease wise symptom and solution page

\section{RESEARCH METHOD}

There are some fundamental steps:

a. Image Acquisition: Images of the infected leaves have taken from the crops. The database has different types images on plant diseases and the images are stored in (.jpg, .png) image format.

b. Image preprocessing: If noises are present in image, interested region in the image is not clear. In the image clipping, smoothing, enhancement is the three steps included in preprocessing phase. The process of image collection and lots of information may bring noise which makes the quality of image dropped. To perform demising different kinds of reduction technique are applicable.

c. Image Segmentation: Image segmentation is the first step and also one of the most critical tasks of image analysis. According to the region of interest, the image will be segmented into different parts.

d. Image Feature Extraction: The features extraction is the input data transform into set of features. The feature set will extract the relevant information. Feature extraction involves simplifying the amount of resources required to describe a large set of data accurately.

e. Image classification: The intent of the classification process is to categorize all pixels in a digital image into one of classes or theme. The objective of image classification is to identify, as a unique gray level (or color), features occurring in an image in terms of the object or type these features actually represent on the ground. Image classification is perhaps the most important part of digital image analysis [12].

\section{BINARY ROBUST INVARIANT SCALABLE KEY POINTS (BRISK): THE METHOD}

Description of the key stages in BRISK, namely feature detection, descriptor composition and key point matching to the level of detail that the motivated reader can understand and reproduce. It is important to note that the modularity of the method allows the use of the BRISK detector in combination with any other key point descriptor and vice versa, optimizing for the desired performance and the task at hand [13].

\subsection{Scale space key point detection}

With the aim of achieving invariance to scale which is crucial for high-quality key points, we go a step further by searching for maxima not only in the image plane, but also in scale-space using the FAST scores as a measure for saliency. Despite discretizing the scale axis at coarser intervals than in alternative high-performance detectors, the BRISK detector estimates the true scale of each key point in the continuous scale-space. In the BRISK framework, the scale-space pyramid layers consist of $n$ octaves $c_{i}$ and $n$ intraoctaves $\mathrm{d}_{\mathrm{i}}$, for $\mathrm{i}=\{0,1 \ldots \mathrm{n}-1\}$ and typically $\mathrm{n}=4$.

The octaves are formed by progressively half-sampling the original image (corresponding $\operatorname{toc}_{0}$ ). Each intra-octave di is located in-between layers $c_{i}$ and $c_{i+1}$ (as illustrated in Figure 5). The first intra-octave do is obtained by down sampling the original image $c_{0}$ by a factor of 1.5 , while the rest of the intra-octave layers are derived by successive half sampling. Therefore, if $t$ denotes scale then $t\left(c_{i}\right)=2$ and $\mathrm{t}\left(\mathrm{d}_{\mathrm{i}}\right)=2^{\mathrm{i}} * 1.5[5]$. 


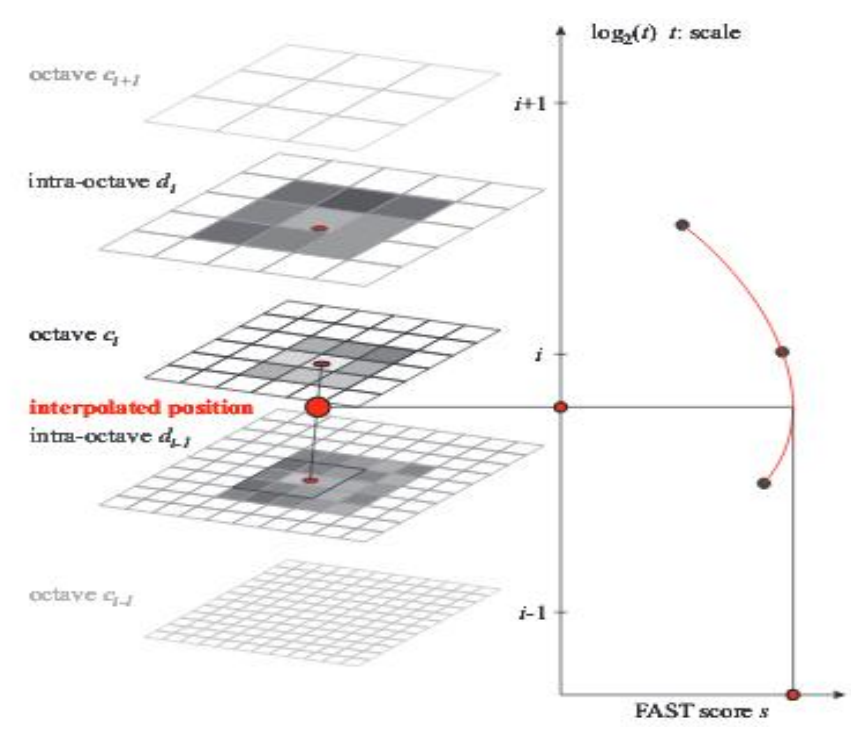

Figure 5. Scale-space interest point detection

Scale-space interest point detection:a key point (i.e. saliency maximum) is identified at octave $c_{i}$ by analyzing the 8 neighboring saliency scores in $\mathrm{c}_{\mathrm{i}}$ as well as in the corresponding scores-patches in the immediately-neighboring layers above and below. In all three layers of interest, the local saliency maximum is sub-pixel refined before a 1D parabola is fitted along the scale-axis to determine the true scale of the key point. The location of the key point is then also re-interpolated between the patch maxima closest to the determined scale [13].

\subsection{Key point description}

Given a set of key points (consisting of sub-pixel refined image locations and associated floatingpoint scale values), the BRISK descriptor is composed as a binary string by concatenating the results of simple brightness comparison tests. This idea has been demonstrated in to be very efficient, however here we employ it in a far more qualitative manner. In BRISK, we identify the characteristic direction of each key point to allow for orientation-normalized descriptors and hence achieve rotation in variance which is key to general robustness. Also, we carefully select the brightness comparisons with the focus on maximizing descriptiveness [13].

\section{DESCRIPTOR MATCHING}

Matching two BRISK descriptors is a simple computation of their Hamming distance as done in BRIEF: the number of bits different in the two descriptors is a measure of their dissimilarity. Notice that the respective operations reduce to a bitwise XOR followed by a bit count, which can both be computed very efficiently on today's architectures [13].

\section{EXPERIMENTAL RESULTS AND DISCUSSION}

7.1. Experimental result with applying algorithmThe experiment is conducted in Android Operating Systems using OpenCv 310 which used image processing and image comparisons techniques with BRISK algorithm. The database is created of six different plants. It consists of 30 images of Paddy plant affected Bacterial Blight Disease, Bacterial Leaf Streak Disease, 20 images of Paddy plants affected by Foot Rot Disease, 38 images of Early Blight and Late Blight of Tomato plant, 38 images of Early Blight and Late Blight of Potato plant, 20 images of Anthracnose Disease of Mango plant and 15 images of Rot Wilt Disease of Jute plant (sample shows in Table 1). 
Table 1. The Sample of different Stages of Affected Crops stored into the Database

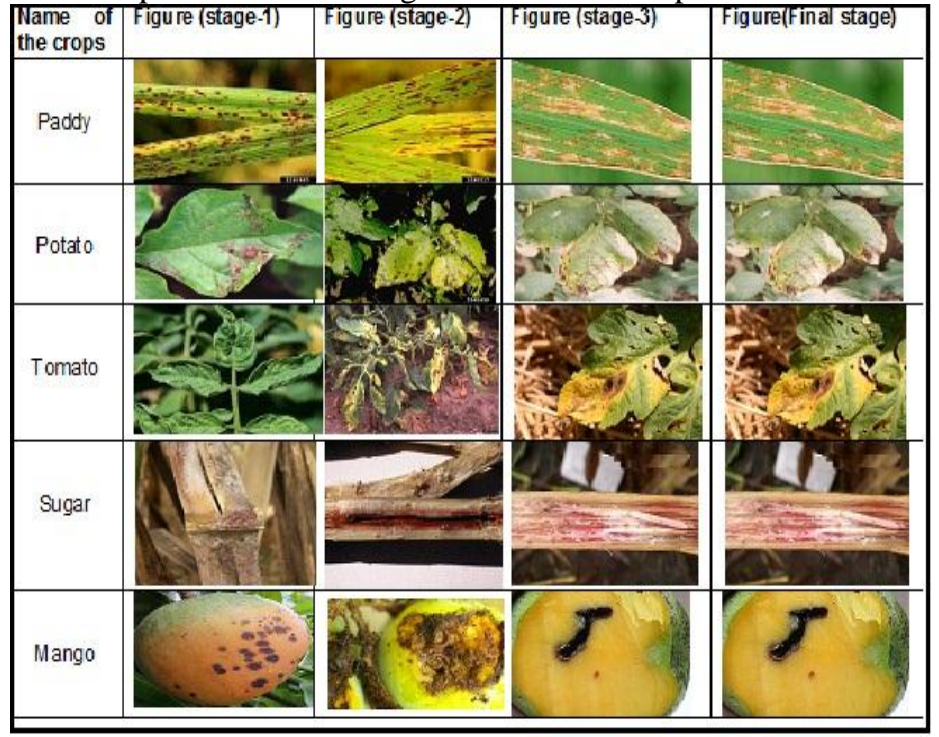

We have used BRISK algorithm in our research to match the uploaded affected crops images with our database images. As per BRISK algorithm's techniques the system takes 100 key objects from both uploaded image e.g. class 1 and all database images e.g. class 2 for the specific crop then it is compare key objects between class 1 and class 2 . After finding the matching of the objects it does calculate the percentage of matched found. It follows the Equation (1):

$$
\text { Percentage (matched) }=\frac{\text { Number of Match found }}{\text { Total number of Objects (key point) }} \mathrm{X} 100
$$

If calculated percentage is above 70 then display the matched image from database and provide the possible disease name and solution about the disease otherwise provides message "Uploaded image is not matched with the database".

\subsection{Result with developed apps}

Menu bar is a user interface with different menu item like Dashboard as Figure 6, Take Photo and Upload Photo etc.

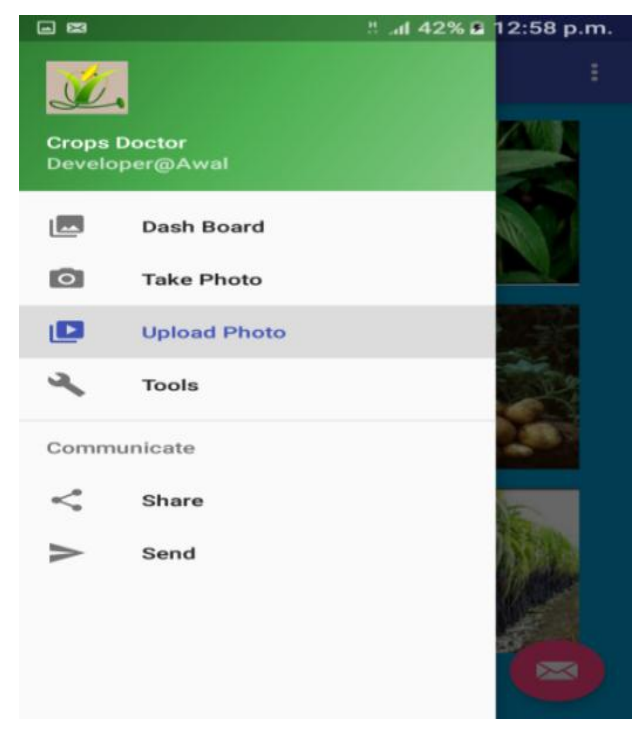

Figure 6. Menu list 
Using Upload Photo menu, farmers can upload affected crops photo to detect diseases and to get solutions. To do it, user has to click Upload Photo menu item, after clicking here users will select specific crops. After clicking Upload button a process dialog will show as Figure 7. If uploaded photo matched with stored images then successful detection result page will be shown with possible disease name, causes and solutions (as illustred in Figure 8)

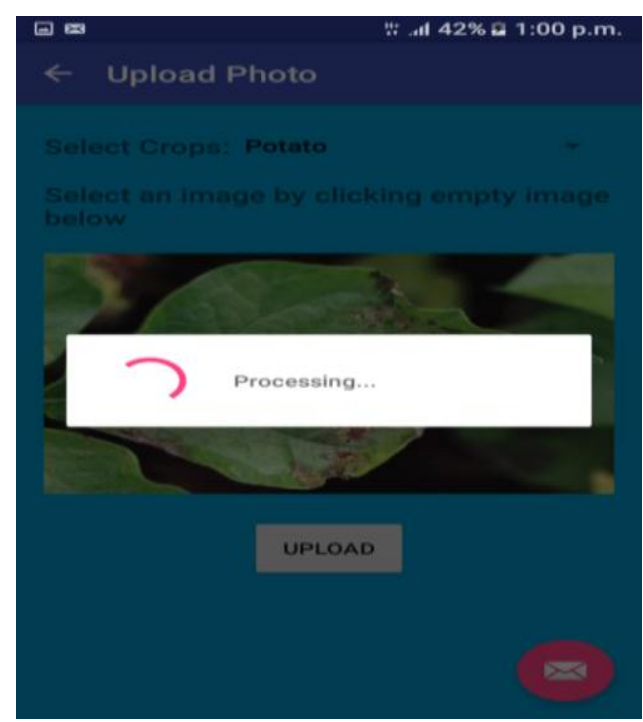

Figure 7. Upload Image Page with process Dialog

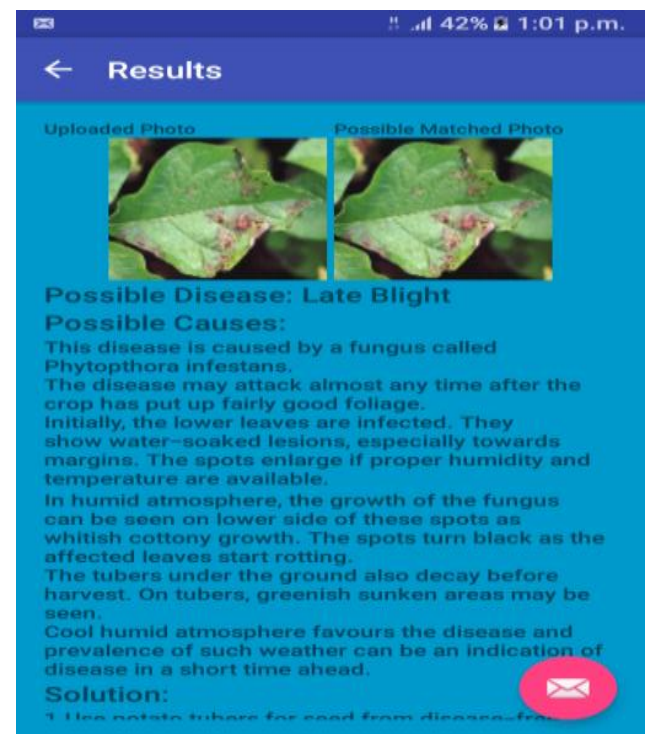

Figure 8. Successful detection result page

If uploaded image does not match with stored image, then it will show "SORRY! Your image is not mathched with our database" message (Figure 9).

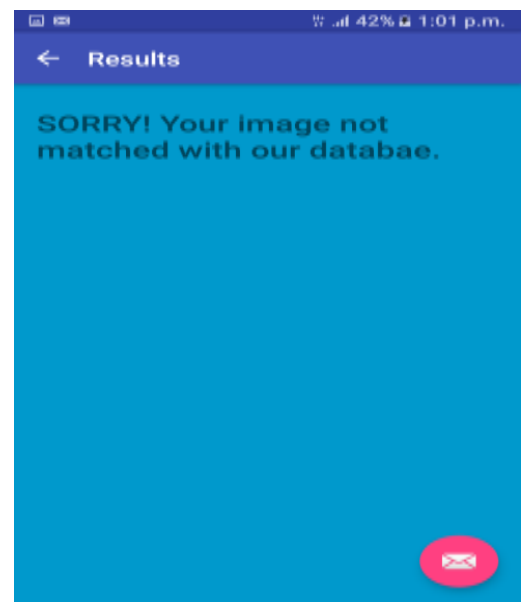

Figure 9. Failed detection result page

\subsection{Comparison statement}

We have compared the Crops Diseases Detection and Solution system with others system and got the better, efficient performance of the system which stated in the Table 2. 
Table 2. Comparison of Crops Diseases Detection and Solution with others Systems

\begin{tabular}{lcccc}
\hline \multicolumn{1}{c}{ Criteria } & $\begin{array}{c}\text { Crops Diseases } \\
\text { Detection and } \\
\text { Solution system } \\
\text { (CDDASS) }\end{array}$ & $\begin{array}{c}\text { Plant Leaf Disease } \\
\text { Identification System } \\
\text { for Android [9] }\end{array}$ & $\begin{array}{c}\text { Detection of Leaf Diseases } \\
\text { and Monitoring the } \\
\text { Agricultural Resources using } \\
\text { Android App [10] }\end{array}$ & $\begin{array}{c}\text { Android Based App to } \\
\text { Prevent Crop Diseased } \\
\text { in Various Seasons [11] }\end{array}$ \\
\hline $\begin{array}{l}\text { Enough information stored } \\
\text { in database about plant }\end{array}$ & Yes & Yes & Yes & No \\
diseases & Yes & No & Yes & Yes \\
$\begin{array}{l}\text { Full part of the diseases } \\
\text { Many kinds of plants }\end{array}$ & Yes & Yes & Yes & No \\
$\begin{array}{l}\text { Easy to use } \\
\text { Accessibility }\end{array}$ & Yes & Yes & Yes \\
Accuracy & Yes & Yes & No & Yes \\
Faster to get result & Yes & No & No & Yes \\
Details solution & Yes & Yes & No & No \\
\hline
\end{tabular}

\section{CONCLUSION}

This Automated Crops Diseases Detection and Solution System is tested in different Android phone and it is working nicely and the system is very useful for farmers especially who are living in rural area and where the agricultural experts are not available. User can easily use this application. Any people can detect and get solution any time using this application. The application is free of cost and does not require any additional device.

\section{REFERENCES}

[1] A. Bhandralia, et al., "Polyhouse Agricultural Marketing System Using Big Data Hadoop," International Journal of Advances in Applied Sciences (IJAAS), vol. 5, pp. 78-84, 2016.

[2] H. Miah, "Agriculture Sector Development Strategy: background paper for preparation of 7th Five Year Plan," http://www.plancomm.gov.bd/7th-five-year-plan/.

[3] H. Sabrol and S. Kumar, "Recognition of Tomato Late Blight by using DWT and Component Analysis," International Journal of Electrical and Computer Engineering (IJECE), vol. 7, pp. 194-199, 2017.

[4] M. S. P. Babu and B. S. Rao, "Leaves Recognition Using Back Propagation Neural Network-Advice For Pest and Disease Control On Crops," India Kisan. Net: Expert Advisory System, 2007.

[5] J. K. Patil and R. Kumar, "Plant Leaf Disease Image Retrieval Using Color Moments," IAES International Journal of Artificial Intelligence (IJ-AI), vol. 2, pp. 36-42, 2013.

[6] B. Li, et al, "Hyperspectral identification of rice diseases and pests based on principal component analysis and probabilistic neural network," Transactions of the CSAE, vol. 5, pp. 143-147, 2009.

[7] H. Jie, "Application of PCA Method on Pest Information Detection of Electronic Nose," IEEE international Conference on Information Acquisition.Weihai, Shandong, China, pp. 1456-1468, 2006.

[8] M. M. Seinl, et al., "Authentications of Myanmar National Registration Card," Indonesian Journal of Electrical Engineering and Informatics (IJEEI), vol. 2, pp. 53-58, 2013.

[9] H. M. Deshmukh, et al., "Plant Leaf Disease Identification System for Android," International Journal of Advanced Research in Computer and Communication Engineering, vol. 5, pp. 396-398, 2016.

[10] S. K. Shekh, et al., "Detection of Leaf Diseases and Monitoring the Agricultural Resources using Android App," International Journal of Innovative Research in Computer and Communication Engineering, vol. 3, pp. 9540-9547, 2015.

[11] S. S. Chavan, et al., "Android Based App to Prevent Crop Diseased in Various Seasons," International Research Journal of Engineering and Technology (IRJET), vol. 3, pp. 1052-1055, 2016.

[12] P. L. Kamble and A. C. Pise, "Review on Agricultural Plant Disease Detection by using Image Processing," International Journal of Latest Trends in Engineering and Technology (IJLTET), vol. 7, pp. 335-339, 2016.

[13] S. Leutenegger, et al., "BRISK: Binary Robust Invariant Scalable Keypoints," IEEE International Conference on Computer Vision, ICCV 2011, Barcelona, Spain, pp. 2548-2555, 2011.

\section{BIOGRAPHIES OF AUTHORS}

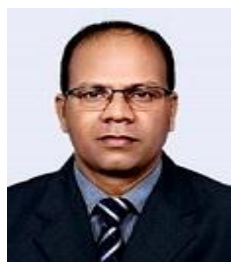

Mohammad Jahangir Alam was born in 1978 in Chittagong. He has completed post-graduation (MSc in CSIT) in 2008 from Southern University Bangladesh, Chittagong. He is the AssistantProfessor at the department of Computer Science and Information Technology in Southern University Bangladesh, Chittagong. He has published many technical papers in reputed national, International Journals and Conferences. 

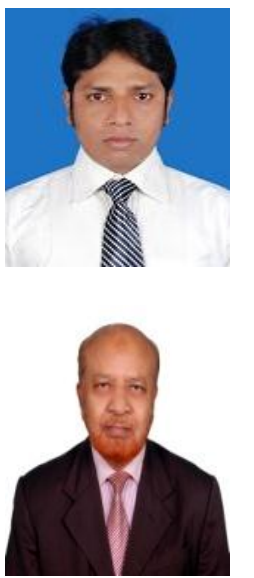

Md. Abdul Awal was born in 1986 in Rajshahi. He has completed his graduation in 2008 from National University, Bangladesh and post-graduation (MSc in CSIT) in 2017 from Southern University Bangladesh, Chittagong. Currently he is working as Senior Software Engineer at Chittagong Container Terminal Management System (CTMS) project in Datasoft Systems Bangladesh Ltd. and has developed several modules of the software being used in CTMS and supports all kinds of software solution as per business need.

Dr. Md. Nurul Mustafa was born on 6th June 1948 at Cox's Bazar, Bangladesh. He has completed his M.Sc. (Physics) in 1970 from University of Chittagong, Bangladesh. He joined as Faculty Member at same Department in 1973.He obtained his higher degree from Concordia University, Montreal, Canada in 1986 and came back and became Professor in 1992. He shifted to Computer Science and Engineering Department as Professor and founder Chairman in 2001 at same University. After retirement from University of Chittagong in 2014, Dr.Mustafa has been working as Professor of Computer Science at Southern University Bangladesh.He published more than fortyfive research articles in the field of Radiation Physics and Computer Science in National and International Journals. 